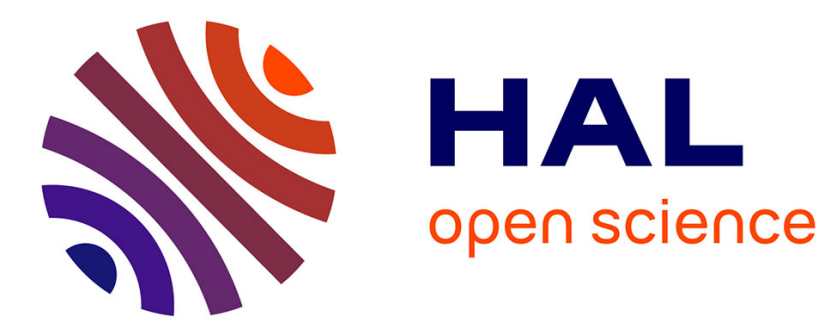

\title{
HRTF measurements of five dummy heads at two distances
}

Adrien Vidal, Philippe Herzog, Christophe Lambourg, Jacques Chatron

\section{To cite this version:}

Adrien Vidal, Philippe Herzog, Christophe Lambourg, Jacques Chatron. HRTF measurements of five dummy heads at two distances. 2021 Immersive and 3D Audio: from Architecture to Automotive (I3DA), Sep 2021, Bologna, Italy. hal-03521905

\section{HAL Id: hal-03521905 \\ https://hal.science/hal-03521905}

Submitted on 11 Jan 2022

HAL is a multi-disciplinary open access archive for the deposit and dissemination of scientific research documents, whether they are published or not. The documents may come from teaching and research institutions in France or abroad, or from public or private research centers.
L'archive ouverte pluridisciplinaire HAL, est destinée au dépôt et à la diffusion de documents scientifiques de niveau recherche, publiés ou non, émanant des établissements d'enseignement et de recherche français ou étrangers, des laboratoires publics ou privés. 


\section{HRTF measurements of five dummy heads at two distances}

\author{
Adrien Vidal \\ Aix Marseille Univ, CNRS, PRISM \\ Marseille, France \\ vidal@prism.cnrs.fr \\ Jacques Chatron \\ Aix Marseille Univ, CNRS, Centrale \\ Marseille, LMA \\ Marseille, France \\ chatron@1ma.cnrs-mrs.fr
}

\author{
Philippe Herzog \\ Arteac-Lab \\ Marseille, France \\ philippe.herzog@arteac-lab.fr
}

\author{
Christophe Lambourg \\ Arteac-Lab \\ Marseille, France \\ christophe.lambourg@arteac-lab.fr
}

\begin{abstract}
Head-Related Transfer Functions (HRTF) characterize the scattering of an incident wave by a listener's head. Measurements of these HRTF is a complex process: the required number of positions is generally high, and the measurement setup could induce a specific scattering (not intended). Especially in the proximity of the listener the source distance has an in influence, and the total number of measurement positions could be superior to a thousand. Measurements of dummy heads are easier than measurements of real listeners, because of the simplified control of the position. However, measurement of dummy heads is challenging, several measurements campaign were done in the literature but not directly comparable because of the variability of experimental setups. In this paper, we present the HRTF measurements of five dummy heads using the same experimental setup. In total, 794 source positions mainly in the half-sphere of positive elevations were measured. These dummy heads were all built to approximate an "average listener", and then should be similar. One of these dummy head was a rigid sphere allowing a comparison to the analytical simulation and deduce an estimation of measurement errors.
\end{abstract}

Keywords—binaural, HRTF measurements, dummy head

\section{INTRODUCTION}

Head-Related Transfer Functions (HRTF) are the transfer functions from sound sources to the listener ears in the free field. These transfer functions are complex, because they depend on the sound source position (azimuth, elevation, distance) and the morphology of the listener (pinnae, head, and torso). HRTF could be computed [1], [2] and also measured. HRTF measurement is a complex process due to the high number of measurement positions, for which a high placement accuracy is required. Moreover, the measurement setup could induce a specific scattering not intended for free field measurements.

Various HRTF measurements were realized during previous works, including measurements of dummy heads [3], of real listeners [4], [5], and in the proximity of the listener [6], [7]. However, all HRTF measurement did not lead to the same results. Indeed, Andreopoulou et al [8] compared the variability of measurements of the same dummy head (Neumann KU100) realized by various institutions at different periods. At total, twelve set of HRTF measured by 10 laboratories were compared. Variability of Interaural Time Difference (ITD) reached $235 \mu \mathrm{s}$, whereas the Just Noticeable Difference (JND) could be between $2 \mu \mathrm{s}$ and $60 \mu \mathrm{s}$ [9]. Differences in spectral amplitudes have also been identified, for frequencies lower than $6 \mathrm{kHz}$ they reached $6.7 \mathrm{~dB}$ for rear incidences and $5 \mathrm{~dB}$ for frontal incidences. Beyond $6 \mathrm{kHz}$, differences of $22 \mathrm{~dB}$ were observed. A similar study was proposed in [10] for five measurements of the dummy head Kemar. Variation in spectral amplitudes reached $4 \mathrm{~dB}$ between $5 \mathrm{kHz}$ and $12 \mathrm{kHz}$. The wider variations observed in this last study, compared to [8], could be explained by a smaller number of campaign measurements. Both works highlighted the fact than HRTF measurements depend on the measurement setup.

The HRTF definition used in this paper is the usual one [11], defined by the following equation:

$$
\operatorname{HRTF}(r, \theta, \phi)=\frac{P_{S}(r, \theta, \phi)}{P_{F F}(r, \theta, \phi)}
$$

Where $P_{S}$ is pressure at the entrance of the ear canal, $P_{F F}$ the pressure at the center of the head while the listener is absent. $r, \theta, \phi$ are the spatial coordinates of the sound source.

In this paper, we present HRTF measurements of five dummy heads at two distances ( $40 \mathrm{~cm}$ and $2 \mathrm{~m}$ ). One of these dummy head was simply a rigid sphere, allowing comparison with analytical computations, allowing to quantify the measurement uncertainties. Dummy heads were built to approximate an "average listener", then measurements should be similar. Thanks to the use of the same setup, measurements can be compared. The measurement protocol is presented in section II, and the post-processing of measurement is presented in section III. The validation of the measurement setup is presented in section IV, and preliminary measurements comparisons are presented in section $\mathrm{V}$.

\section{MEASUREMENT PROTOCOL}

The measurement itself consisted in measurement of impulse response of sound sources at different points in space with the five dummy heads.

\section{A. Dummy heads}

Five dummy heads were characterized in these works:

- Gras KEMAR 45 BB-3 (Sound Quality Recording), with large pinnae (VA-style, model KB0090 and KB0091). Microphones inside the head are Gras 40AG (1/2 inch) with preamplifiers 26AS and a conditioning amplifier Nexus 2690. This dummy head is named "Kemar" in the following.

- $\quad$ Bruel \& Kjaer 4100D. Microphones inside the head are $B \& K \quad 4189$ (1/2 inch) and the preamplifiers are $B \& K$ 2671. This dummy head is named " $B \& K$ " in the following. 
- Cortex MK2. Microphones inside the head are MK $231 \mathrm{E}$, connected to isemCOM SA-P48/CCP-C adaptors. This dummy head is named "Cortex".

- Head Acoustics HRS-II.2. We do not know the type of microphones inside the head. None of the possible equalization filter was selected. This dummy head is named "Head Acoustics"

- A sphere was built in ABS by $3 \mathrm{D}$ printing. It consists of two half spheres (4 mm thick walls), stiffened by ribs and sealing compound. The exterior radius is $8.75 \mathrm{~cm}$, and a location for $1 / 4$ inch microphones has been provided on each side so that microphones are placed with an incident angle of $100^{\circ}$ with the median plane. Microphone Gras 40PR were used for the measurements. This dummy head is named "Sphere".

Pictures of these five dummy heads are shown by Fig. 1 . The point between the two microphones was considered as the center of the head. This point is quite easily identifiable, and corresponds to the definition proposed by the ITU [12]. For the sphere, the center of the head was its center, which is not exactly the point between the two microphones (placed slightly on the back). Each dummy head was placed at the center of the setup using a cross laser. The precision of such a setup is about $5 \mathrm{~mm}$. The dummy head was placed on a turn table, allowing to measure several azimuths for one loudspeaker position. The turn table was configured to rotate the dummy head with a step of $5^{\circ}$, leading to 72 azimuths positions.

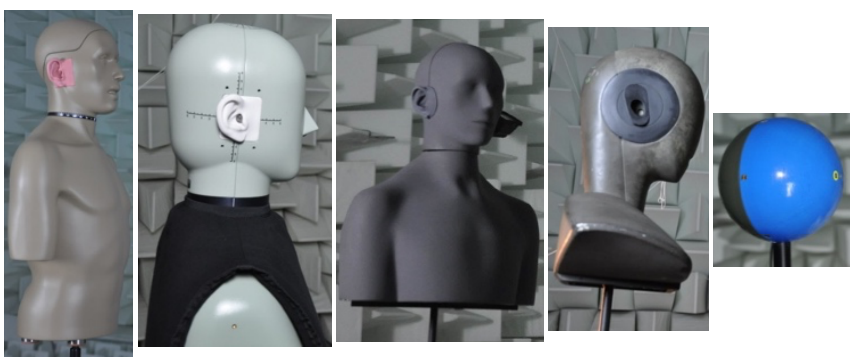

Fig. 1. Pictures of the five dummy heads. From left to right: Kemar, B\&K, Cortex, Head Acoustics, Sphere

\section{B. Hardware and software}

HRTF were measured in an anechoic chamber with dimensions $5.3 \times 6.0 \times 5.0 \mathrm{~m}^{3}$, its wall covered by $80 \mathrm{~cm}$ thick porous wedges (about $150 \mathrm{~Hz}$ cutoff frequency and $25 \mathrm{dBA}$ background noise). Input/output signals were controlled by a soundcard MOTU 24I/O. The dummy head was placed on a turn table LT360 EX, with a resolution of $1^{\circ}$. A Max/MSP interface allowed to control the whole operations (rotation of the table, reading and recording of signals). The signal played through the loudspeakers was an exponential sweep ranging from $50 \mathrm{~Hz}$ to $20 \mathrm{kHz}$ during $6.1 \mathrm{~s}$, computed from the method presented in [13]. Both the raw measured sweep and the impulse response deduced from the measured sweep were saved in 24 bits wav format at a sampling frequency of 48 $\mathrm{kHz}$.

\section{Loudspeaker setups}

HRTF were measured at two distances, and two different setups were used according to the measurement distance.
Both measurements were realized in the same anechoic chamber, only the loudspeakers setup was different.

\section{1) Setup for $2 \mathrm{~m}$ measurements}

For $2 \mathrm{~m}$ measurements, an array of loudspeakers Meyer Sound MM-4X placed in two arcs around the dummy heads was used. Only the arc placed in the median plane was used (six loudspeakers placed at elevations $0^{\circ}, 15^{\circ}, 30^{\circ}, 45^{\circ} 60^{\circ}$ and $90^{\circ}$ ), as illustrated in Fig. 2. For each loudspeaker 72 azimuthal head positions were characterized, excepted for the $90^{\circ}$ elevation (only one measurement). Then a total of 361 measurements were realized with this setup.
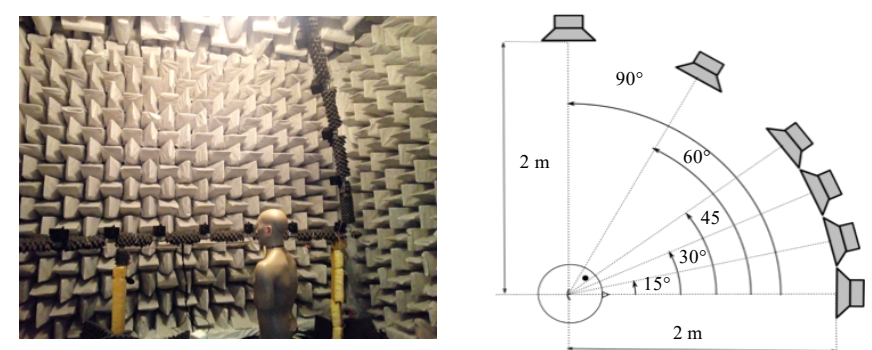

Fig. 2. Illustration of the setup for the $2 \mathrm{~m}$ measurements. Left: picture with the Kemar dummy head; right: sketch of the setup

\section{2) Setup for $40 \mathrm{~cm}$ measurements}

For the $40 \mathrm{~cm}$ measurements, a specific sound source was designed to approximate a point sound source with a low frequency response adequate for our measurements. This sound source was a 3.3" loudspeaker (Visaton FRS 8M) mounted in a 1.2 liter closed box as illustrated by Fig. 3 . The input signal was amplified with a Trends TA 10.1. This sound source was placed at seven different elevations: $-30^{\circ},-15^{\circ}$, $0^{\circ}, 15^{\circ}, 30^{\circ}, 60^{\circ}$ and $90^{\circ}$. As for $2 \mathrm{~m}$ measurements, for each loudspeaker position 72 azimuthal head positions were characterized, excepted for the $90^{\circ}$ elevation (only one measurement). A total of 433 measurements were realized with this setup.

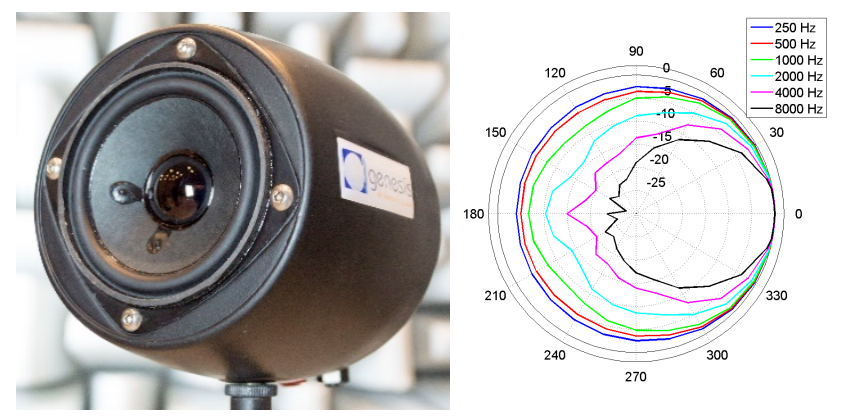

Fig. 3. Left: picture of the sound source used for $40 \mathrm{~cm}$ measurements; Right: directivity diagram of this sound source

\section{Free-field measurements}

A part of the estimation of HRTF is the measurement of the pressure at the center of the setup. Transfer functions between loudspeakers and a microphone placed at the center of the setup were recorded. A free field microphone (B\&K 4190) pointing toward each loudspeaker was used. The Frequency Response Functions (FRF) are shown by Fig. 4. FRF measured with loudspeakers placed at $2 \mathrm{~m}$ were quite similar to each other, the most different FRF from the others was the one measured with the loudspeaker placed at $90^{\circ}$. For 
this FRF, there was a high number of oscillations probably due to comb filtering caused by reflections on the turn table. These oscillations caused deviations up to $10 \mathrm{~dB}$ from other FRF, mainly in the $[3.5 \mathrm{kHz}-8 \mathrm{kHz}]$ frequency band. This variability was not problematic for the HRTF estimation since it was compensated according to the equation (1). The bandwidth at $+/-10 \mathrm{~dB}$ was $[150 \mathrm{~Hz}-18 \mathrm{kHz}]$, then adequate for our measurements. For the loudspeaker used at $40 \mathrm{~cm}$, the bandwidth is $[200 \mathrm{~Hz}-18 \mathrm{kHz}]$ at $+/-10 \mathrm{~dB}$, which is also adequate for the targeted measurements.

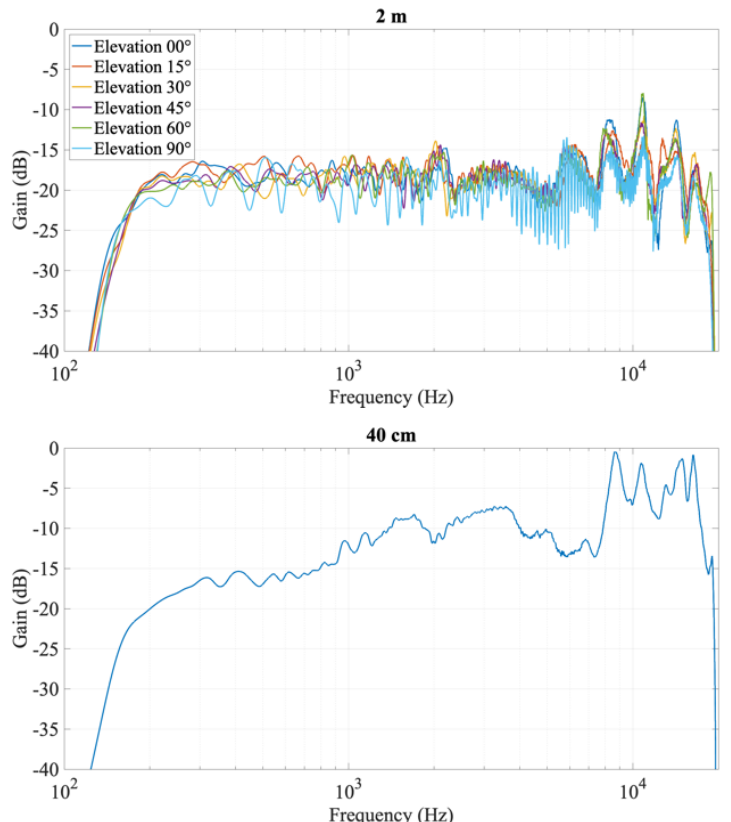

Fig. 4. Up: Frequency Response Functions of the six loudspeakers used for the $2 \mathrm{~m}$ measurements (placed at elevations from $0^{\circ}$ to $90^{\circ}$ ); bottom: FRF of the loudspeaker used for the $40 \mathrm{~cm}$ measurements

\section{POST-PROCESSING OF HRTF}

A few post-processing operations were realized to get HRTF corresponding to the definition (1) and to minimize biases linked to the measurement protocol. Four operations were realized, detailed hereafter.

\section{A. Temporal windowing}

The first operation consisted in a temporal windowing of impulse responses, to limit their length and to suppress possible echoes due to the measurement setup. A dual-band windowing was processed, to shorten the higher frequencies more than the lower frequencies. First, a crossover filter has been designed with a cutoff frequency of $700 \mathrm{~Hz}$. At lower frequencies $(<700 \mathrm{~Hz})$, a $43 \mathrm{~ms}$ window was applied. At higher frequencies $(>700 \mathrm{~Hz})$, a $7.4 \mathrm{~ms}$ window was applied, and samples of the last $1 \mathrm{~ms}$ were apodised with a half Hann window. Fig. 5 reports windowed and non-windowed Impulse Response measured at $2 \mathrm{~m}$ for a free-field measurement (without dummy head). Fig. 6 is the equivalent of Fig. 5 in the frequency domain. Effect of windowing is equivalent to a smoothing, in the temporal domain peaks after $7 \mathrm{~ms}$ are smoothed, and in the frequency domain comb filtering is attenuated mainly between $800 \mathrm{~Hz}$ and $5 \mathrm{kHz}$.

\section{B. Free-field normalisation}

The second step was the normalization of dummy heads transfer functions by the free-field measurement (without dummy head). This operation allowed to compensate all the

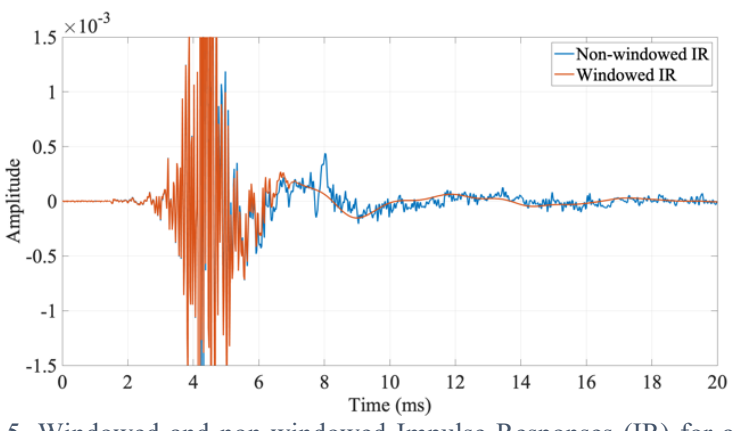

Fig. 5. Windowed and non-windowed Impulse Responses (IR) for a freefield measurement (without dummy head)

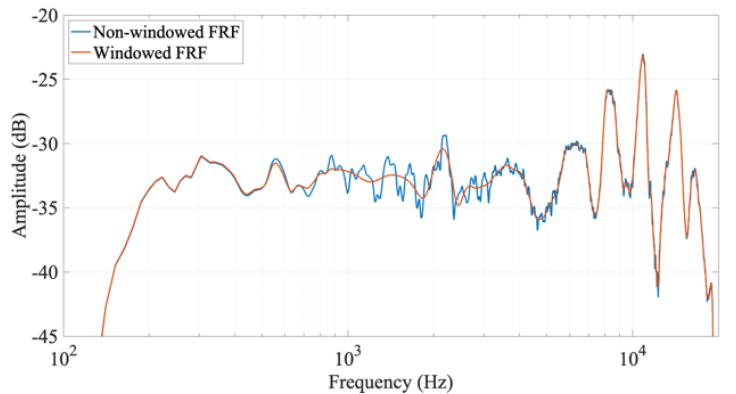

Fig. 6. Windowed and non-windowed Frequency Response Functions (FRF) for a free-field measurement (without dummy head)

contributions which are not linked to the spatial position, such as the sound source response. This operation was realized by dividing the complex transfer functions. Note that the ear toward the sound source is closer to it than the microphone at the center of the setup. The free-field normalization then induces a negative time of arrival, and Impulse Responses associated to these HRTF may not systematically be causal.

\section{High and low frequencies processing}

Sounds sources have a limited bandwidth. Outside their bandwidths, the transfer function estimation is noisy and not accurate. The HRTF estimate is thus indeterminate, which must be coped with. This is the case at higher and lower frequencies and is processed according to the following steps.

At lower frequencies, measured values are not fundamental for HRTF estimation: below $250 \mathrm{~Hz}$ wavelengths are larger than $1.4 \mathrm{~m}$, which is larger than all dimensions of characterized dummy heads. Therefore, variations of HRTF are marginal at these low frequencies. Moreover, perceptual localization of sound sources at lower frequencies is mainly due to phase differences directly related to ear positions. We consider that microphone positions of all the characterized dummy heads are similar, so the phase at lower frequencies should be similar too.

To deal with the low frequency problem, a few approaches were proposed in the literature. Below $103 \mathrm{~Hz}$, Brinkmann [14] fixed the amplitude of HRTF at $0 \mathrm{~dB}$ while maintaining the measured phase. Other authors [15] used a modelization method for frequencies below $400 \mathrm{~Hz}$, but the method was not presented in the paper. We assume that level differences might occur according to the source incidence, and a method based on simple model seemed preferable to an arbitrary fixed amplitude. For frequencies lower than $230 \mathrm{~Hz}$, HRTF were computed from a spherical head model with a $8.75 \mathrm{~cm}$ radius and receivers placed on the diameter. A crossover filter $\left(12^{\text {th }}\right.$ order Linkwitz-Riley) with a cutoff 
frequency of $230 \mathrm{~Hz}$ was designed to combine computed and measured data.

An example of the low frequency modelization is presented in Fig. 7. For the area around the cutoff frequency $(230 \mathrm{~Hz})$, the post-processed HRTF is smooth and corresponds to the combination of computed and measured HRTF. Below $150 \mathrm{~Hz}$ the amplitude of the computed HRTF is constant and about $3 \mathrm{~dB}$, which is significantly different from $0 \mathrm{~dB}$ as proposed by other authors.

Higher frequencies were simply suppressed using a 12-order Linkwitz-Riley low-pass filter with a cutoff frequency of 19 $\mathrm{kHz}$.

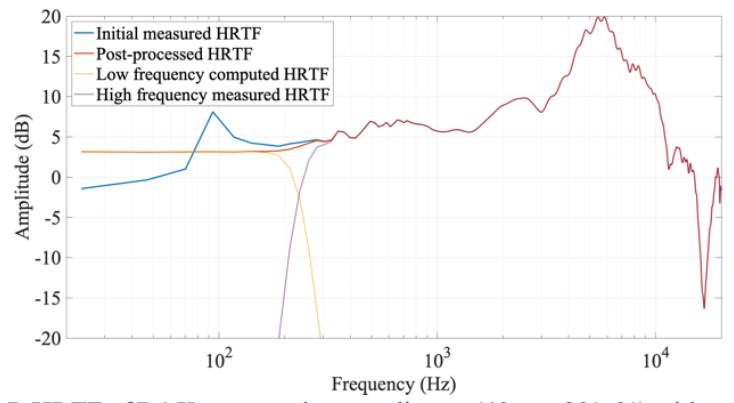

Fig. 7. HRTF of B\&K measured at coordinates $\left(40 \mathrm{~cm}, 90^{\circ}, 0^{\circ}\right)$ without (blue line) and with (red line) low frequency processing

\section{HRTF equalization}

The final processing step was HRTF equalization. This equalization aimed at compensating the microphone response, and potential measurement artifacts independent from the direction. HRTF can be equalized in two ways [16]: from a HRTF with a given incidence (usually the frontal incidence), or from a diffuse-field HRTF. Diffuse-field equalization has several advantage in comparison to freefield equalization [16], and was preferred in this work.

Obviously, the diffuse-field HRTF measurement requires a diffuse field as in a reverberant room. We did not have access to such a facility, so the diffuse-field HRTF was estimated from a weighted average of measurements at various angles. Because of the non-uniform repartition of our measurement points, a weighting was applied, taking into account the solid angle related to each measurement position. To estimate this solid angle, a spherical Voronoi diagram was used [17]. Since our measurement setup was not a complete sphere and lacked measurements at lower elevations, this method assigns an excessive weight to measurements at the lowest elevation, which we had to compensate manually. For $40 \mathrm{~cm}$ measurement, we attributed the same weight for measurements at $-30^{\circ}$ elevation and for $30^{\circ}$ measurements. For $2 \mathrm{~m}$ measurements, we attributed the same weight for measurements at $0^{\circ}$ elevation and for $15^{\circ}$ measurements. Attributed weights are reported in the TABLE I for the $40 \mathrm{~cm}$ measurements and in the TABLE II for the $2 \mathrm{~m}$ measurements. For both cases, weight at elevation $90^{\circ}$ is significantly higher than others. However, it concerns only one measurement whereas there are 72 azimuths for all other elevations.

TABLE I. Weights attributed to $40 \mathrm{~cm}$ measurements according to the source elevation

\begin{tabular}{|c|c|c|c|c|c|c|c|}
\hline Elevation $\left(^{\circ}\right)$ & -30 & -15 & 0 & 15 & 30 & 60 & 90 \\
\hline Weight & 1.30 & 0.45 & 0.45 & 0.45 & 1.30 & 1.95 & 6.67 \\
\hline
\end{tabular}

TABLE II. Weights attributed to $2 \mathrm{~m}$ measurements according to the source elevation

\begin{tabular}{|c|c|c|c|c|c|c|}
\hline Elevation $\left(^{\circ}\right)$ & 0 & 15 & 30 & 45 & 60 & 90 \\
\hline Weight & 0.80 & 0.80 & 0.74 & 0.65 & 1.86 & 11.84 \\
\hline
\end{tabular}

The estimation of the diffuse-field HRTF is shown by Fig. 8. Diffuse field HRTF are similar for all dummy heads: amplitude is around $0 \mathrm{~dB}$ until $1 \mathrm{kHz}$, then between $1 \mathrm{kHz}$ and $7 \mathrm{kHz}$ there is an amplification area, and beyond $7 \mathrm{kHz}$ there is an attenuation zone. Some variations may however be noticed according to the dummy head, such as the localization of the maximum amplitude. There are also slight differences between the estimation at $40 \mathrm{~cm}$ and $2 \mathrm{~m}$ : between $300 \mathrm{~Hz}$ and $500 \mathrm{~Hz}$, successive peaks of around $1 \mathrm{~dB}$ for all dummy heads are visible at $40 \mathrm{~cm}$ but not at $2 \mathrm{~m}$. Similarly, there is a systematic notch around $2.2 \mathrm{kHz}$ for measurements at $2 \mathrm{~m}$. This could be linked to the measurement setup.

For each diffuse-field HRTF, an associated inverse FIR filter $H(f)$ was computed from the following equation:

$$
H=\left[C^{*} C+\beta\right]^{-1} \cdot\left[C^{*}(A-C I d)\right]+I d
$$

$C$ is the diffuse-field HRTF to equalize, $\beta$ is a Tikhonov regularization parameter with a value of $10^{-\frac{50}{20}}, A$ the Fourier transform of a pure delay with a length of 1024 samples, Id a unitary vector and $*$ is the conjugate operator. The minimal phase component was extracted using the cepstral method [18] and the FIR filter obtained as the inverse Fourier transform. As an example, the diffuse-field HRTF estimated for $2 \mathrm{~m}$ measurements of the dummy head B\&K and its associated filter of are shown by Fig. 9 .
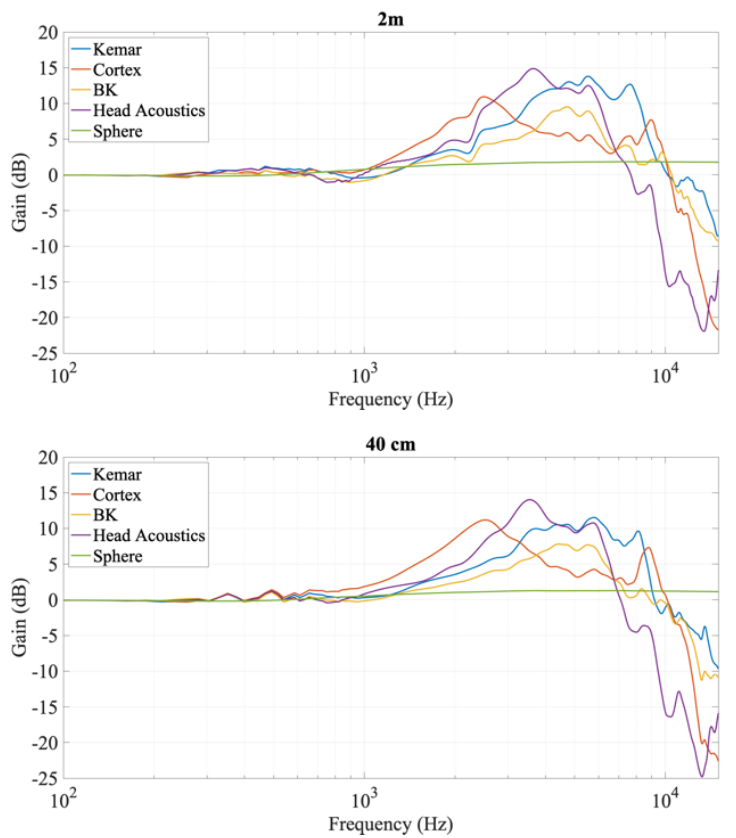

Fig. 8. Estimation of diffuse-field HRTF for the five dummy heads at $2 \mathrm{~m}$ (top) and at $40 \mathrm{~cm}$ (bottom) 


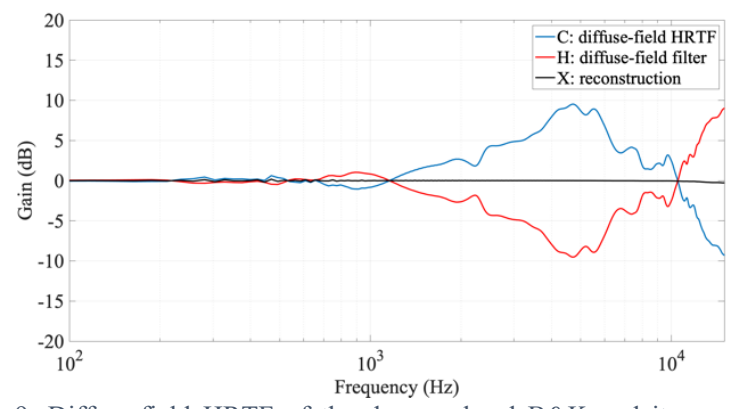

Fig. 9. Diffuse-field HRTF of the dummy head B\&K and its associated inverse filter. The black line ("reconstruction") is a simulation

\section{VALIDATION OF THE MEASUREMENT SETUP}

In this part, some preliminary analysis is made to check if measurements correspond to expectation: the setup could have induced unwanted scattering, spatial positions could have been wrong, and directivity of the sound source in the proximity of the listener could have influenced the measurement.

\section{A. Sphere: measures vs model}

HRTF measured with the sphere are compared with analytical computations. Measured and computed HRTF for a few source incidences are reported in Fig. 10. Measured HRTF are very close to the computed ones with few differences, detailed hereafter.

At $40 \mathrm{~cm}$, differences are lower than $2 \mathrm{~dB}$ for the four represented HRTF up to $8 \mathrm{kHz}$. There are slight oscillations on measured HRTF, while computed HRTF are smooth. Between $350 \mathrm{~Hz}$ and $600 \mathrm{~Hz}$ and between $900 \mathrm{~Hz}$ and $2 \mathrm{kHz}$, amplitude of measured HRTF are underestimated by about 1 $\mathrm{dB}$ for incidences $0^{\circ}, 180^{\circ}$ and $270^{\circ}$. Around $2.3 \mathrm{kHz}$, there is a systematic peak, reaching $1 \mathrm{~dB}$ for the $90^{\circ}$ incidence. These oscillations look like comb filtering, caused by reflections whose effects depend on the specific locations of source and microphone. For $0^{\circ}$ incidence at higher frequencies $(>11 \mathrm{kHz})$, amplitude of measured HRTF is significantly lower than the computed HRTF: the difference reaches $7 \mathrm{~dB}$ at $13 \mathrm{kHz}$ and $9 \mathrm{~dB}$ at $14 \mathrm{kHz}$. This difference could be explained by the directivity of the loudspeaker: for the $0^{\circ}$ incidence, microphones were farther from the loudspeaker axis. However, source directivity should lead to a similar difference for the $180^{\circ}$ incidence while measured and computed HRTF are then very close.

At $2 \mathrm{~m}$, there are also systematic differences: between 800 $\mathrm{Hz}$ and $1800 \mathrm{~Hz}$, measured HRTF are attenuated by about 1 $\mathrm{dB}$ compared to the computed ones. At $270^{\circ}$ incidence the minimum amplitude is not located at the same frequency: it is around $8 \mathrm{kHz}$ for the computed HRTF and $7.5 \mathrm{kHz}$ for the measured one. This leads to differences up to $3 \mathrm{~dB}$ between $6 \mathrm{kHz}$ and $8 \mathrm{kHz}$. Oscillations appear mainly beyond $2 \mathrm{kHz}$ for all incidences. Differences were not the same than for 40 $\mathrm{cm}$ measurements, thus confirming the hypothesis of reflections causing comb filtering.

\section{B. Left-right asymetry}

Dummy heads are supposed to be symmetric, and HRTF measured with the left ear should be the same as the HRTF measured with the right ear. Observed differences could be either related to dummy heads asymmetry, or to the inaccuracy of the measurement setup (or both). Asymmetry

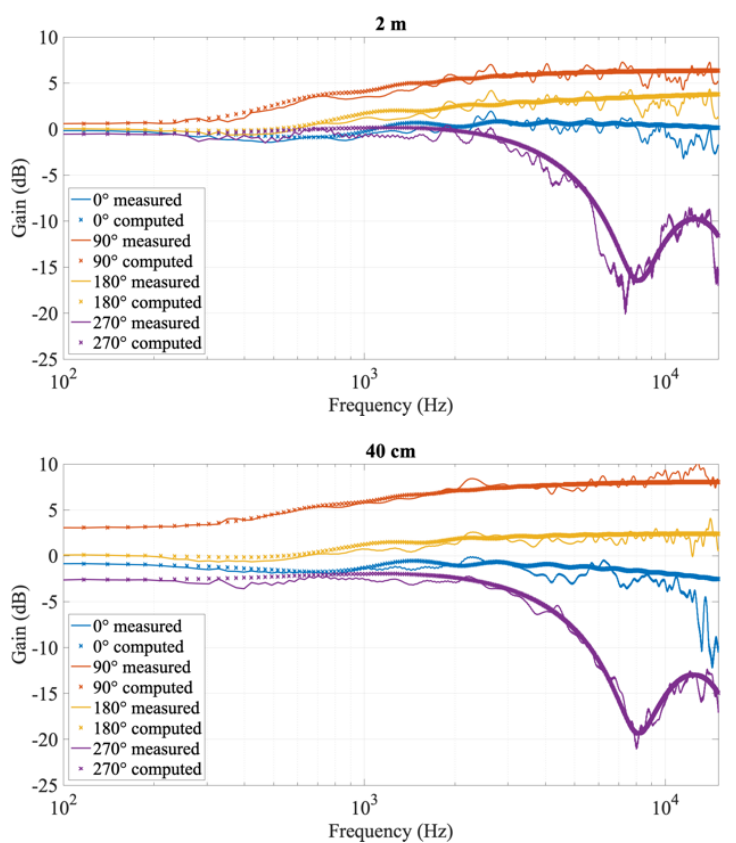

Fig. 10. Measured and computed HRTF of a rigid sphere for a few source incidences in the horizontal plane at $2 \mathrm{~m}$ (top) and $40 \mathrm{~cm}$ (bottom)

of a dummy head (Neumann KU-100) was observed in previous works [8], estimated with a metric proposed by [19]. This metric is a correlation distance computed according to the Equivalent Rectangular Bandwidth (ERB) [20].

We used the same metric $d$ defined by the following equation for an ERB band $N$ :

$$
d(\theta, \phi, N)=1-\frac{\left|\sum_{k=1}^{K} H_{L}\left(\theta, \phi, f_{k}\right) \cdot H_{R}^{*}\left(360-\theta, \phi, f_{k}\right)\right|}{\sqrt{\sum_{k=1}^{K} H_{L}^{2}\left(\theta, \phi, f_{k}\right) \cdot H_{R}^{2}\left(360-\theta, \phi, f_{k}\right)}}
$$

With $\mathrm{H}_{\mathrm{L}}\left(\theta, \phi, \mathrm{f}_{\mathrm{k}}\right)$ and $\mathrm{H}_{\mathrm{R}}\left(360-\theta, \phi, \mathrm{f}_{\mathrm{k}}\right)$ left and right HRTF at incidences $(\theta, \phi)$ and $(360-\theta, \phi)$ at the frequency $f_{k}$. The dissymmetry of the Kemar dummy head for the $2 \mathrm{~m}$ distance and $0^{\circ}$ elevation is reported in the Fig. 11. The asymmetry is not equivalent for the two half-circle around the dummy head: for ipsilateral incidences (azimuth $<180^{\circ}$ ) the asymmetry is inferior to 0.05 until $10 \mathrm{kHz}$. Beyond $10 \mathrm{kHz}$, asymmetry is higher and reach 0.2 . For contralateral incidences, three different area are identifiable. The first one concerns azimuths between $180^{\circ}$ and $240^{\circ}$ for which asymmetry is close to the one for ipsilateral incidences. The second area concerns azimuths between $240^{\circ}$ and $300^{\circ}$, for which asymmetry is high at frequencies higher than 2 $\mathrm{kHz}$. The third area concerns azimuths higher than $300^{\circ}$ for which asymmetry is low at all frequencies.

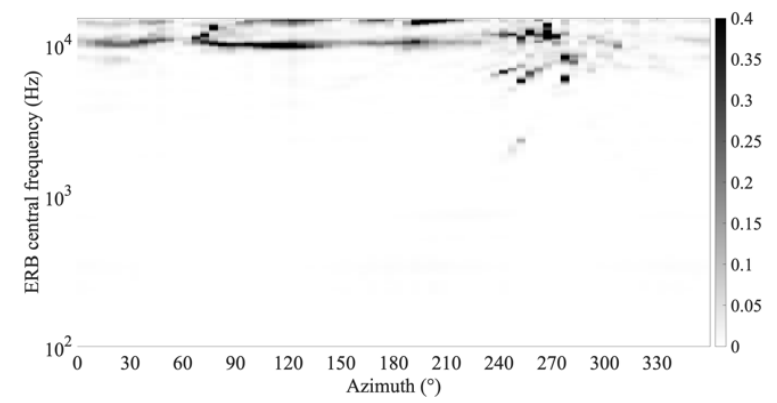

Fig. 11. Left/right asymmetry of the Kemar dummy head, computed for $2 \mathrm{~m}$ measurements in the horizontal plane 
The difference between ipsilateral and contralateral incidences could be explained by a lower signal to noise ratio for contralateral incidences related to the head diffraction. The quasi-systematic asymmetry observed at $10 \mathrm{kHz}$ could be related to pinnae difference. The associated wavelength is about $3 \mathrm{~cm}$, which corresponds to pinnae dimensions. In a previous study [8] using the same metric, asymmetry of about 0.25 was observed in the ipsilateral area for frequencies lower than $9 \mathrm{kHz}$. In the contralateral area, asymmetry reaching 0.4 were observed. Values of asymmetry are similar in our works, allowing us to validate the measurement setup.

Fig. 12 represents left and right HRTF of the Kemar dummy head in the horizontal plane for source incidences of $250^{\circ}$ and $110^{\circ}$ respectively. According to observations made on Fig. 11, it is a particularly asymmetrical case. The observed notch around $2.3 \mathrm{kHz}$ is less pronounced for the right ear, leading to a local difference of $8 \mathrm{~dB}$. Between $5 \mathrm{kHz}$ and $9 \mathrm{kHz}$, amplitude variations are less pronounced for the right ear, leading to deviations of about $5 \mathrm{~dB}$. Beyond $9 \mathrm{kHz}$, the alternation between peaks and notches is shifted for the two ears, leading to deviations exceeding $10 \mathrm{~dB}$. Despite these deviations, both measurements are reasonably similar.

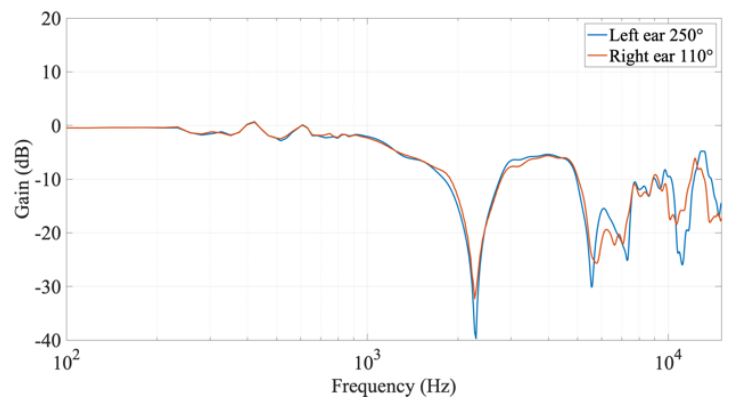

Fig. 12. HRTF of the Kemar dummy head measured on the left ear for an incidence of $250^{\circ}$ and on the right ear for an incidence of $110^{\circ}$

\section{COMPARISON OF MEASUREMENTS}

In this section, a preliminary analysis of results is made to compare both the effect of the measurement distance and the dummy head.

\section{A. Magnitude of HRTF in the horizontal plane}

The Fig. 13 represents the magnitude of HRTF measured on the left ear at the two distances for the five dummy heads in the horizontal plane. In general, HRTF look like each other. Especially for frequencies higher than $1 \mathrm{kHz}$, there is an amplification area (for azimuths $<180^{\circ}$ ) or an attenuation area (azimuths $>180^{\circ}$ ). In the attenuation area around $270^{\circ}$, there is also a slight amplification area: it could corresponds to the "bright spot" [21] which corresponds to an arrival in phase of several acoustic waves. This bright spot could be observed for all dummy heads at both distances.

The distance effect is visible for all dummy heads. The dynamic between the amplification area and the attenuation area differs according to the distance. In general, differences between extrema of the two distances is about $5 \mathrm{~dB}$. As an example, at lower frequencies $(<800 \mathrm{~Hz})$, magnitude of ipsilateral incidences is about $5 \mathrm{~dB}$ at $40 \mathrm{~cm}$, whereas it is about $0 \mathrm{~dB}$ at $2 \mathrm{~m}$.

HRTF look like each other, but it is possible to differentiate them. In particular, the bright spot is different according to the dummy head. The sphere is a special case, since its bright spot goes until $15 \mathrm{kHz}$, whereas it is not clearly identifiable beyond $3 \mathrm{kHz}$ for other dummy heads. It could be explained by the simplicity of the spherical model: due to its symmetry, several waves arrive in phase at the ear. On the contrary, the complex morphology of other dummy heads limits the number of in phase arrivals. At lower frequencies, wavelengths are larger than the dimensions of the morphologic details, and waves could arrive in phase at the ear. Excepting the bright spot, magnitude repartition according to the frequency and the azimuth differs only slightly between all dummy heads.

\section{B. Interaural Time Differences}

The Interaural Time Difference (ITD) is frequently used in binaural technology. ITD can be computed in many different ways, 32 methods were compared in a previous study [22]. Large variations exceeding $200 \mu$ s were observed according to the method. However, authors did not favor a method over another. In this work, we used a method based on an onset threshold detection to estimate the arrival time on each ear. The ITD is then the difference in arrival time. The threshold used here is $-12 \mathrm{~dB}$, because it seemed more stable with our measurement. ITD measured in the horizontal plane at the two distances for the five dummy heads is shown by Fig. 14. Differences between dummy heads are slight: the sphere is the most different from the other especially for the measurement at $2 \mathrm{~m}$. Difference reaches $60 \mu$ s between the ITD of the Sphere and the ITD of Kemar for the $90^{\circ}$ azimuth. At $40 \mathrm{~cm}$, the maximal ITD is around $80^{\circ}$ for Kemar, whereas it is located around $90^{\circ}$ for other dummy heads. There is no clear effect of the measurement distance on the ITD.

\section{CONCLUSION}

In this paper we presented HRTF measurements of five dummy heads at two distances $(40 \mathrm{~cm}$ and $2 \mathrm{~m})$. We presented the measurement setup and the post-processing operations (windowing, normalization, processing of low and high frequencies, diffuse-field equalization). One of these dummy heads was a 3D-printed rigid sphere, for which the numerical computation was possible. The comparison between measured and computed HRTF from the sphere allowed to quantify the measurement inaccuracies, which were acceptable. Asymmetry between measurements of the two ears of dummy heads were in the same range than a previous study. Both observations make it possible to validate our measurement setup. In the last section, we presented preliminary observations to compare the dummy heads and the effect of distance on measurements. As a perspective of these work, a more detailed comparison could be conducted to possibly regroup dummy heads. It requires to use a specific metric to establish a distance between HRTF. Measured HRTF could also be compared to equivalent measurements in other institutions. Another perspective concerns a perceptive evaluation of this database. A listening test could be conducted to check if listeners are able or not to differentiate audio content build with the HRTF of the different dummy heads, all supposed to approximate an "average" listener. 

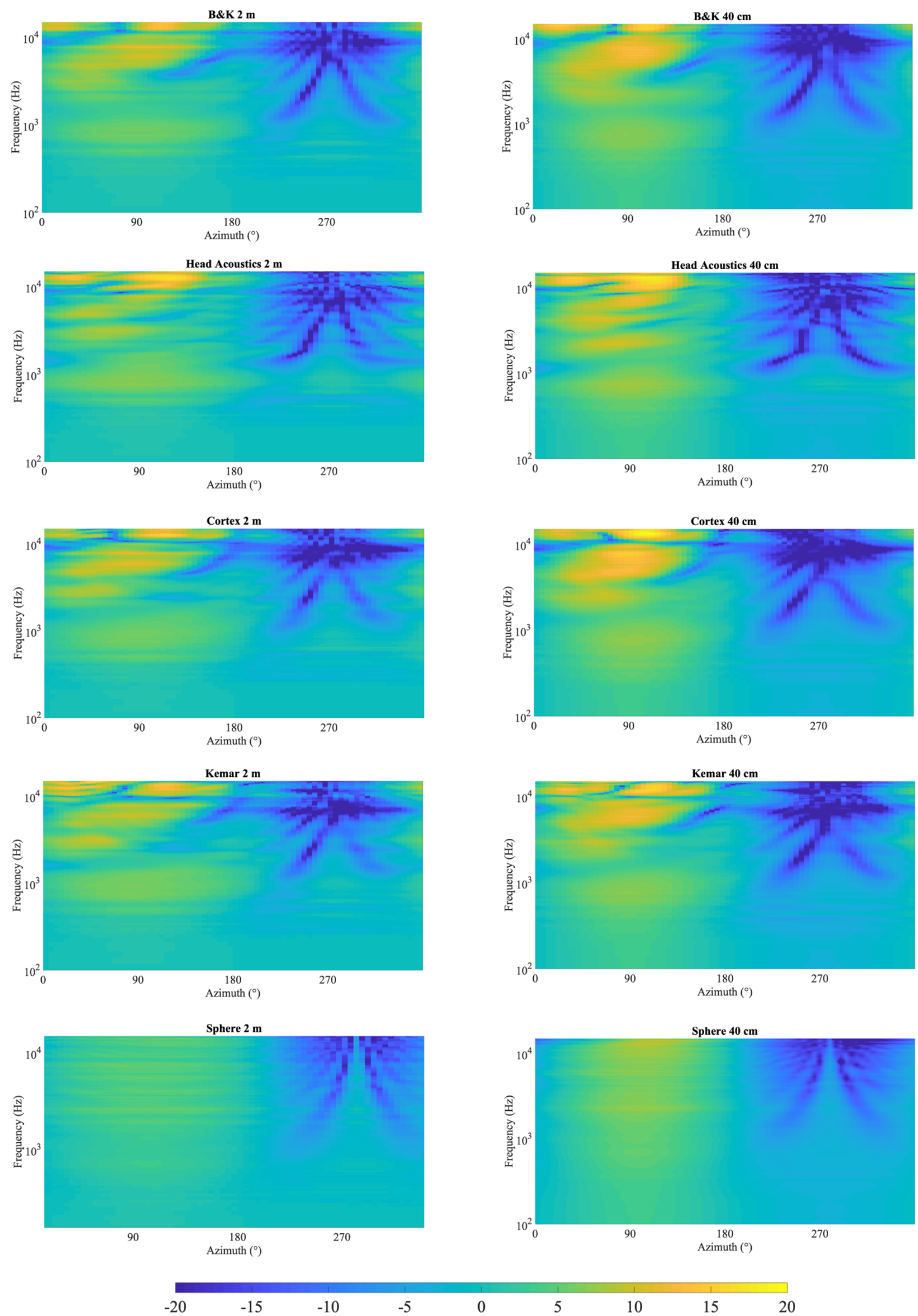

Fig. 13. Magnitude (in dB) of measured HRTF in the horizontal plane of the five dummy heads at $2 \mathrm{~m}$ (left figures) and at $40 \mathrm{~cm}$ (right figures). From top to bottom: B\&K, Head Acoustics, Cortex, Kemar, Sphere. 


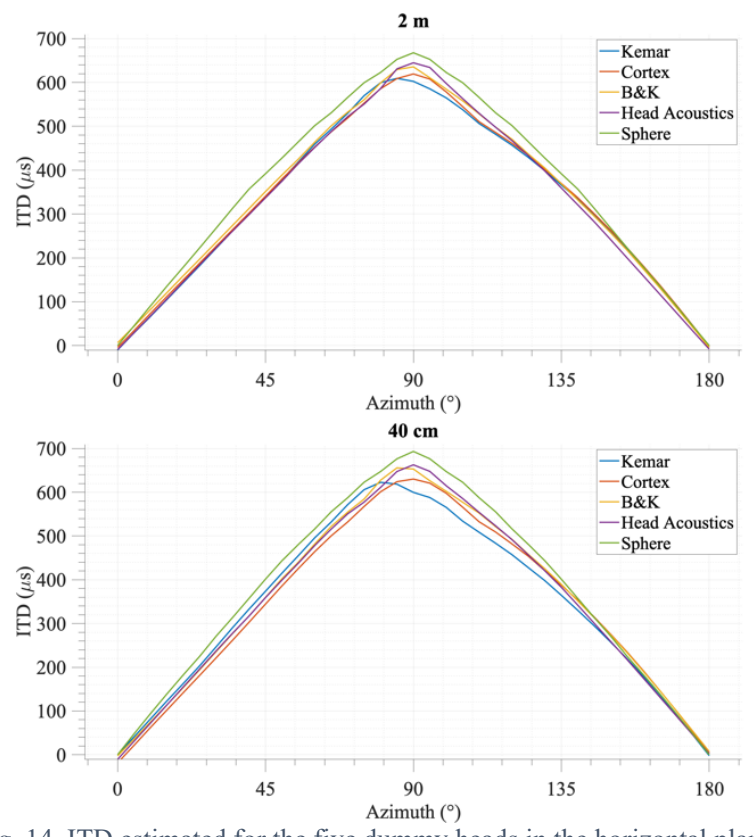

Fig. 14. ITD estimated for the five dummy heads in the horizontal plane at 2 $\mathrm{m}$ (up) and $40 \mathrm{~cm}$ (bottom)

\section{ACKNOWLEDGMENT}

This project was funded by the ANR (ANR-11-BS09001). The authors would like to thank Guy Rabau for his help to make support of dummy heads and Vincent Long for the 3D-printing of the sphere.

\section{REFERENCES}

[1] B. Katz, "Boundary element method calculation of individual head-related transfer function. I. Rigid model calculation," Journal of the American Society of America, vol. 110, no. 5, pp. 2440-2448, 2001

[2] T. Huttunen et al., "Rapid generation of personalized HRTFs," presented at the AES 55th International Conference, Helsinki (Finland), 2014

[3] W. G. Gardner and K. Martin, "HRTF measurements of a KEMAR dummy head microphone," Media Lab Perceptual Computing, MIT, Technical Report, 1994.

[4] R. Algazi, R. Duda, D. Thompson, and C. Avendano, "The CIPIC HRTF database," presented at the IEEE Workshop on applications of Signal Processing to Audio and Acoustics, New York (USA), 2001

[5] O. Warusfel, "Listen HRTF database," 2003 http://recherche.ircam.fr/equipes/salles/listen/index.html

[6] D. Brungart, W. Rabinowitz, and N. Durlach, "Auditory localization of nearby sources. II - Head-related transfer functions," Journal of the American Society of America, vol. 106, no. 3, pp. 1465-1479, 1999.

[7] H. Wierstorf, M. Geier, A. Raake, and S. Spors, "A free database of Head-Related Impulse Response measurements in the horizontal plane with multiple distances," presented at the 130th AES Convention, London (UK), 2011.

[8] A. Andreopoulou, D. R. Begault, and B. Katz, "Inter-laboratory round robin HRTF measurement comparison," IEEE Journal of selected topics in signal processing, vol. 9, no. 5, pp. 895-906, 2015.

[9] J. Blauert, Spatial hearing: the psychophysics of human sound localization, revised edition. 1997.

[10] X.-L. Zhong, X. Xu, and B.-S. Xie, "Auditory consistency of HRTF of KEMAR from different databases," presented at the 23rd International Congress on Sound and Vibration, Athens (Greece), 2016.

[11] H. Moller, "Fundamentals of binaural technology," Applied Acoustics, vol. 36, no. 3/4, pp. 171-218, 1992.

[12] ITU-T P.58, "Head and torso simulators for telephonometry," International Telecommunication Union, 2013.

[13] A. Novak, L. Simon, and P. Lotton, "Nonlinear system identification using exponential swept-sine signal," IEEE Transactions on Instrumentation and Measurement, vol. 59, no. 8, pp. 2220-2229, 2009.

[14] F. Brinkmann, A. Lindau, S. Weinzierl, G. Geissler, and S. van de Par, "A high resolution head-related transfer function database including different orientations of head above torso," presented at the AIA-DAGA Conference on Acoustics, Merano (Italia), 2013.

[15] G.-Z. Yu, B.-S. Xie, and D. Rao, "Characteristics of near-field head-related transfer function for KEMAR," presented at the AES 40th International Conference, Tokyo (Japan), 2010.

[16] V. Larcher, J.-M. Jot, and G. Vandernoot, "Equalization methods in binaural technology," presented at the 105th Audio Engineering Society Convention, San Francisco (USA), Sep. 1998.

[17] H. S. Na, C. N. Lee, and O. Cheong, "Voronoi diagrams on the sphere," Computational Geometry, vol. 23, no. 2, pp. 183-194, 2002.

[18] A. Oppenheim and R. Schafer, Digital signal processing (pp 337345), Prentice-Hall international Editions. 1975.

[19] X.-L. Zhong, F.-C. Zhang, and B.-S. Xie, "On the spatial symmetry of Head-Related Transfer Functions," Applied Acoustics, vol. 74, no. 6, pp. 856-864, 2013.

[20] B. C. Moore and B. R. Glasberg, "A revision of Zwicker's loudness model," Acta Acustica united with Acustica, vol. 82, pp. 335-345, 1996.

[21] R. Duda and W. Martens, "Range dependence of the response of a spherical head model," Journal of the American Society of America, vol. 104, no. 5, pp. 3048-3058, 1998.

[22] B. Katz and M. Noistering, "A comparative study of interaural time delay estimation methods," Journal of the American Society of America, vol. 135, no. 6, pp. 3530-3540, 2014. 